\title{
Betrachtung der Kostenfaktoren bei der Erdgasversorgung im liberalisierten Markt Österreichs aus der Sicht des Vertriebs als Energiedienstleister für Wien
}

Bilanzgruppensystem; Energiedienstleister; Entflechtung; Erdgasmarkt; Erdgasvertrieb; Kostenbetrachtung; Liberalisierung

Die Umwälzungen im österreichischen Erdgasmarkt in den Jahren nach der völligen Öffnung der Märkte im Oktober 2002 führten zu einer inzwischen sehr starken Entflechtung der Vertriebs- und Netzfunktion. Dies bedeutet auch für den Erdgasvertrieb in öffentlicher Hand eine Neuorientierung bei der Betrachtung der relevanten Kostensituation und der davon beeinflussten Marktposition nicht nur gegenüber anderen Erdgasanbietern, sondern vorwiegend auch mit Blick auf die klassischen Konkurrenzenergien, wie Erdöl, Fernwärme oder erneuerbare Energieträger. Trotz der Auftrennung der zuvor integrierten Versorgungsfunktionen muss der Vertrieb als Energiedienstleister nach wie vor den Blick auf die für den Endkunden anfallenden Gesamtkosten wahren, um im Markt erfolgreich bleiben zu können.

\section{Allgemein}

Der österreichische Erdgasmarkt erlebte in den Jahren seit der 100\%igen Marktöffnung im Jahr 2002 einen massiven Umwälzungsprozess. Die traditionellen Strukturen, die von integrierten Erdgasversorgern in den einzelnen Bundesländern gekennzeichnet waren, wurden aufgebrochen und somit ein neues, wettbewerbs- und kundenorientiertes System unter Aufsicht einer Regulationsbehörde geschaffen. Ausschlaggebend für diese Entwicklungen waren die Liberalisierungsbestrebungen der EU, deren erklärtes Ziel die Auflösung nationaler oder regionaler Monopole war (und ist). Im Oktober 2002 kam es mit der Umsetzung der entsprechenden EU-Richtlinien in der Form des österreichischen Gaswirtschaftsgesetzes (GWG, BGBl. I Nr. 121/2000, zuletzt geändert durch BGBl. I Nr. 148/2002) zur Trennung von Netzbetrieb (=natürliches Monopol) und Vertriebsaktivitäten (Markt). Dieser Umstand bedingt auch im Bereich des Erdgasvertriebs an Endkunden eine völlig neue Sichtweise, die eine tiefer gehende Analyse - insbesondere aus energiewirtschaftlicher Perspektive - erforderlich macht, wobei hier Crastan folgend der Vertrieb als Energiedienstleister und Motor des liberalisierten Marktes verstanden wird (Crastan 2009, S. 125 ff. und insbesondere S. 131ff.). Anders als der Vertrieb im Elektrizitätsmarkt hat der Erdgasvertrieb allerdings das Augenmerk besonders auf übergreifende Betrachtungen zu legen, da der Energieträger Erdgas nach wie vor im intensiven Wettbewerb mit Konkurrenzenergieträgern, wie Erdöl, Fernwärme oder erneuerbaren Energien steht. Vertraut man den offiziellen Daten zum Wechselverhalten im österreichischen Erdgasmarkt (AGGM 2009), die sehr geringe Kunden- 
wechselzahlen und damit Wettbewerbsaktivität zeigen (die Bedeutung der Wechselbereitschaft von Endkunden für den Erdgasvertrieb wurde in der wissenschaftlichen Diskussion bereits untersucht (Wiedmann/Hennings/Kilian 2005, S. 44ff.)), so ist gerade die Position im Vergleich zu anderen Energieformen für den wirtschaftlichen Erfolg von Erdgas ausschlaggebend. Aus der Sicht des Energiedienstleisters sollen in dieser Betrachtung sowohl die netz- als auch die energieseitigen Einflussfaktoren auf die Erdgasbeschaffung, die Beschaffungskosten und damit auf die gegenüber dem Endkunden darstellbaren Gesamtabgabepreise aus der Sicht eines typischen großstädtischen Energievertriebs (hier am konkreten Beispiel des Wiener Versorgers) eingeschlossen werden. Um eine möglichst umfassende Übersicht bieten zu können, werden auch die sonstigen Beeinflussungen der Gesamtkostensituation, wie vor allem Steuern, Abgaben oder Messpreise, berücksichtigt. Der Fokus soll aber vorwiegend auf der (energiewirtschaftlichen) Kostenseite liegen; die bei Versorgern naturgemäß angestellten Überlegungen zur Festlegung von Marketing- und Vertriebskosten, Personalkosten oder Margen in den einzelnen Kundensegmenten werden hier nur in der Form von geschätzten Zuschlägen berücksichtigt, um eine durchgängige Darstellung bieten zu können.

\section{Das österreichische Marktmodell}

Mit der vollständigen Marktöffnung zum 1.10.2002 wurde der österreichische Gasmarkt in drei Regelzonen eingeteilt (Ost, Tirol und Vorarlberg). In jeder dieser Regelzonen sorgt der Regelzonenführer für die technische Stabilität des Transportleitungssystems und die Verwaltung bestehender Transportkapazitäten im Leitungsnetz (Abbildung 1). 


\section{Die 3 Regelzonen des österreichischen Gasmarktes}

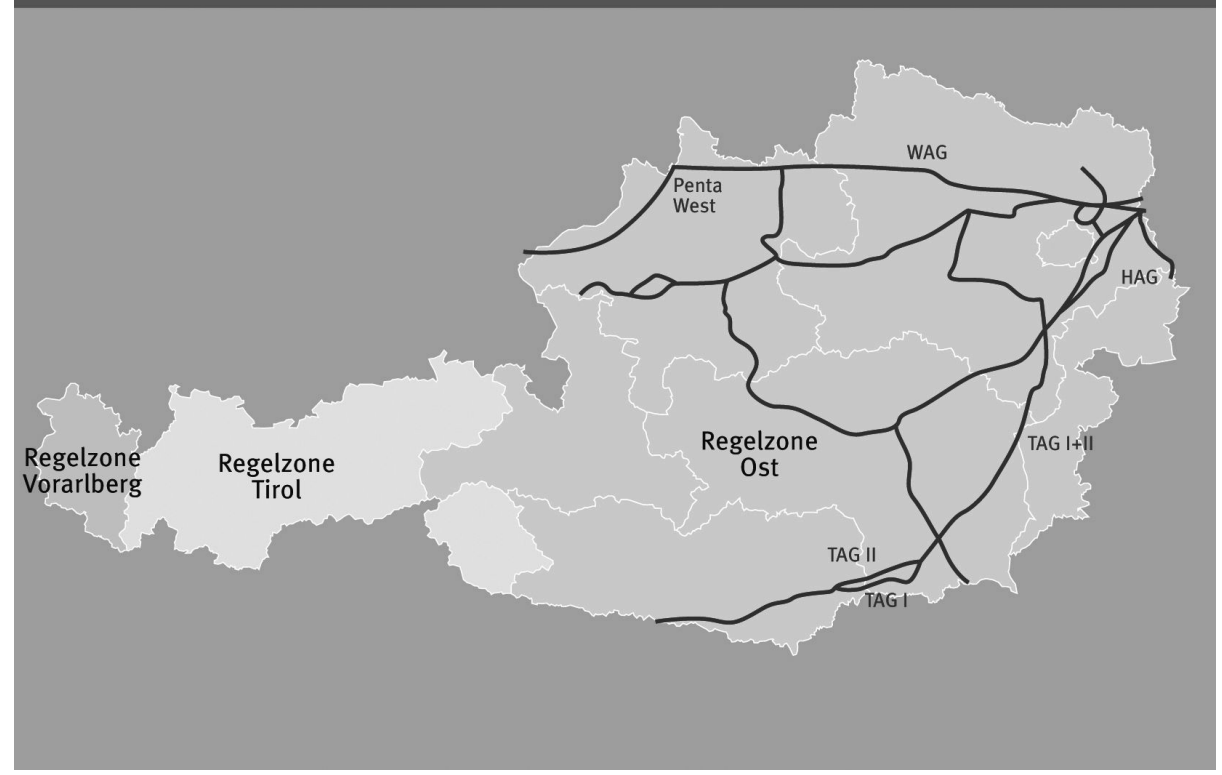

Abb. 1: Die drei Regelzonen des österreichischen Erdgasmarkts

Quelle: Eigene Darstellung

In den einzelnen Regelzonen sind die verschiedenen Marktteilnehmer, wie Versorger, Händler und Kunden zu Gruppen („Bilanzgruppen“) zusammengeschlossen, die für den Ausgleich zwischen Aufbringung und Entnahme von Erdgas verantwortlich sind. Das Bilanzgruppenmodell ist die Voraussetzung für eine erfolgreiche Trennung von monopolistischem Netzbereich und dem Handelsbereich. Diese Trennung ist erforderlich, um den Wettbewerb zwischen Versorgern zu ermöglichen und den vormals an ihren Gebietsversorger gebundenen Kunden die Chance zu bieten, Energie auch bei anderen Anbietern zu beziehen. Durch die Netzbereiche übergreifenden Bilanzgruppen können neue Versorger Kunden aus allen Bereichen der Regelzone zusammenfassen und beliefern (Abbildung 2). 


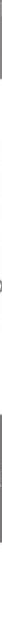

Beziehung der Marktteilnehmer im österreichischen Erdgasmarkt

gene Darstellung

ng:

betreiber sind Betreiber von Fern- und Verteilungsnetzen.

lzonenführer sind verantwortlich für die Systemstabilität der Regelzone.

ringstelle (Bilanzgruppenkoordinator oder Market Operator): ermittelt anhand Netzbetreibern und Marktteilnehmern zur Verfügung gestellten Daten die Diff chen Ein- und Ausspeisung (das entspricht der Ausgleichsenergiemenge), den $\mathrm{F}$ usgleichsenergie und verwaltet die einzelnen Marktteilnehmer in organisatorisc chnungstechnischer Hinsicht.

steht für die einzelnen Verbrauchsstellen (Zählpunkte), also die Endkunden.

Zweck von Bilanzgruppen besteht hauptsächlich in der Sicherstellung des Ausglei rauch und Beschaffung von Erdgas in der Bilanzgruppe. Die Bilanzgruppe plant der Bilanzgruppenmitglieder für jede Stunde und gemeinsam mit den Versor kung der Nachfrage. Weichen die benötigten Mengen vom prognostizierten Volu dafür Ausgleichsenergie vom Regelzonenführer abgerufen werden, die über eine r geschaffenen Ausgleichsenergiemarkt gehandelt wird. Die Bilanzgruppen bzy n Rahmen tätigen Lieferanten sind für die Energiebeschaffung ünd die Versorg 
ende Analyse bezieht sich auf den Kleinkunden-, Gewerbe- und Kleinindustriesel Strom- und Wärmeerzeugungsbereich sowie industrielle Großkunden werden n tigt. Der grundlegende Aufbau der Kundensegmentierung folgt den Vorgaben c nutzungstarife-Verordnung (Gas-Systemnutzungstarife-Verordnung, zuletzt SNT-VO 2008-Novelle 2009), die eine mengen- bzw. verbrauchsabhängige St Ermittlung von Netzentgelten vorsieht - ein auch im Energiebereich sinnvoller ung 3).

\section{denanalyse Wien}

\begin{tabular}{|c|c|c|c|c|c|c|c|c|}
\hline \multicolumn{4}{|c|}{ Basisdaten } & \multicolumn{4}{|c|}{ Energie } & \\
\hline Anzahl & Verbrauch & $\begin{array}{l}\text { So/Wi- } \\
\text { Verhältnis }\end{array}$ & $\begin{array}{c}\text { Anschluss- } \\
\text { wert }\end{array}$ & Volumen & $\begin{array}{l}\text { Maximal- } \\
\text { leistung }\end{array}$ & $\begin{array}{l}\text { Band- } \\
\text { leistung }\end{array}$ & $\begin{array}{l}\text { Speicher- } \\
\text { leistung }\end{array}$ & $\begin{array}{l}\text { 12-Monats- } \\
\emptyset \text {-Leistung }\end{array}$ \\
\hline & in $M W h$ & in $\%$ & in $M W$ & in $M W h$ & in $M W$ & in $M W$ & in $M W$ & in $M W$ \\
\hline 525.055 & 1.721 .309 & $62,07 \%$ & 4.672 & 207.705 & 1.086 & 196 & 889 & 893 \\
\hline 339.044 & 4.476 .697 & $78,45 \%$ & 4.443 & 1.273 .620 & 1.496 & 511 & 985 & 923 \\
\hline 84.059 & 2.330 .854 & $79,11 \%$ & 1.324 & 678.511 & 775 & 266 & 509 & 479 \\
\hline 17.427 & 1.000 .925 & $79,76 \%$ & 333 & 297.875 & 333 & 114 & 219 & 206 \\
\hline 6.324 & 834.964 & $79,00 \%$ & 214 & 242.140 & 278 & 95 & 183 & 172 \\
\hline 2.558 & 767.336 & $78,31 \%$ & 214 & 217.256 & 255 & 88 & 168 & 158 \\
\hline 1.565 & 1.020 .577 & $77,58 \%$ & 267 & 281.475 & 327 & 117 & 211 & 202 \\
\hline 427 & 983.352 & $73,60 \%$ & 180 & 232.071 & 264 & 112 & 152 & 187 \\
\hline 976.458 & 13.136 .014 & & 11.646 & 3.430 .653 & 4.815 & 1.500 & 3.315 & 3.219 \\
\hline
\end{tabular}

Kundenanalyse Wien

gene Darstellung

ungen:

hl bezeichnet die Anzahl der Zählpunkte in den einzelnen Staffeln; ein Zählp net jede Einspeise- und/oder Entnahmestelle, an der eine Gasmenge messtechnisc registriert wird.

rauch ist die jährliche Abgabemenge an alle Zählpunkte.

Sommer-/Winterverhältnis ist ein Indikator für die Verteilung der Last über $d$ ei hier nur der Winterantell angegeben wurde. Ein Verhâltnis von 50:50 charak jelsweise einen Kunden mit bandförmiger Abnabme, üblicherweise einen indu 
Kunden mit kontinuierlicher Produktion. Der in Wien vorherrschende Typ ist hingegen wesentlich stärker heizlastig - ein großer Teil der Jahresmenge wird im Winter bezogen.

- Der Anschlusswert bezeichnet die technisch mögliche Maximalleistung.

- Die angeführten Gaszählergrößen geben nur typische Größenklassen in der jeweiligen Staffel an; selbstverständlich kommen auch noch größere Zähler zum Einsatz, wie auch im Punkt „Messkosten“" noch ausführlicher behandelt wird.

Die Kenngrößen für Energie und Netz werden in den entsprechenden Unterpunkten näher behandelt.

\section{Transportkosten - Netz}

Neben der Trennung von Netz- und Energiebereich ist die Einführung eines durch Verordnung festgesetzten Tarifs, der an die Stelle der früher in den Erdgasbezugs- oder separaten Transportverträgen vereinbarten Entgelte getreten ist, die wesentlichste Neuerung in der Beschaffungslandschaft für Erdgas. Der Transportkunde (d. h. Netznutzer) zahlt für die von ihm benötigte Dienstleistung ein Entgelt an den Netzbetreiber, an dessen Netz seine Anlage(n) angeschlossen ist bzw. sind. Dieses beinhaltet alle Transportkosten vom Eintrittspunkt (Entry-Point) in das österreichische Netz über die Fern- und Verteilerleitungsebene bis zum Hausanschluss. Anders als z. B. in Deutschland wurde in Österreich für die Festlegung von Tarifen auf das Briefmarkensystem gesetzt. Das bedeutet, dass weder ein Entry-Exit-Modell, noch eine transaktionskostenorientierte Entgeltermittlung erfolgt. Einerseits fallen also für die Buchung von Kapazitäten an den Eintrittspunkten in das österreichische Netz keine Kosten an, andererseits spielt auch die Entfernung des Verbrauchspunktes zu diesen Punkten keine Rolle. Die gesamten Kosten des übergeordneten Fernleitungssystems (Ebene 1) werden zusammengefasst und entsprechend der leistungs- und arbeitsmäßigen Belastung auf die einzelnen Netzbereiche verteilt. Auf diese Weise wird das gedankliche Modell eines „Gassees“ in die Realität umgesetzt, was im Gegensatz zu distanzabhängigen Tarifmodellen aber naturgemäß zu deutlichen Verzerrungen und Benachteiligungen von nahe an den Einspeisepunkten gelegenen und leistungsstarken Netzbereichen führt. Aus diesem Grund werden die endgültig den Netzen zugeordneten Kosten noch mit Ausgleichsfaktoren korrigiert, die sich am Anteil der Fernleitungskosten an den gesamten Leistungskosten des Verteilnetzbetreibers und der Entfernung von den Entry Points orientieren. Diese Faktoren sollen dafür Sorge tragen, dass die Veränderungen zum System vor der Liberalisierung (und damit die Widerstände gegen die Neuregelung) nicht zu groß werden. Die Kosten der Fernleitungsebene werden den originären Kosten der Verteilernetze hinzugerechnet und somit Bestandteil der Verteilerbriefmarke der einzelnen Netzbereiche. Die gesamten Kosten des Netzbereiches inklusive der vorgelagerten Fernleitungsebene, der Systemsteuerung und der Regulation werden in einem verordneten Briefmarkentarif abgebildet - das Ergebnis ist ein 100\% Exittarif in Form eines einheitlichen Entgelts für jeden Kunden (Abbildung 4). 
$0-8.000$

$001-15.000$

$.001-40.000$

$.001-80.000$

$001-200.000$

$.001-400.000$

$001-1.107 .000$

$-5.000 .000$

$001-10.000 .000$

$001-100.000 .000$

100.000 .001

\begin{tabular}{|l|l|}
\hline Zone 1 & 1,3746 \\
\hline Zone 2 & 1,1455 \\
\hline Zone 3 & 1,1455 \\
\hline Zone 4 & 0,7741 \\
\hline Zone 5 & 0,7741 \\
\hline Zone 6 & 0,7741 \\
\hline Zone 7 & 0,5306 \\
\hline
\end{tabular}

\begin{tabular}{|c|c|}
\hline Zone A & 0,2506 \\
\hline Zone B & 0,2020 \\
\hline Zone C & 0,1120 \\
\hline Zone D & 0,1120 \\
\hline
\end{tabular}

\begin{tabular}{|c|c|}
\hline Staffel 1 & 250 \\
\hline Staffel 2 & 250 \\
\hline Staffel 3 & 250 \\
\hline Staffel 4 & 250 \\
\hline Staffel 5 & 250 \\
\hline Staffel 6 & 250 \\
\hline Staffel 7 & 250 \\
\hline Staffel A & \\
\hline Staffel B & \\
\hline Staffel C & \\
\hline Staffel D & \\
\hline
\end{tabular}

Netzentgelt Ebene 3 im Netzbereich Wien

SNT-VO 2008-Novelle 2009

werden auf Grund ihrer Größe und der davon abgeleiteten Pflicht zur Leistungsn ieden. Ab einem Jahresverbrauch von $400.000 \mathrm{kWh}$ ist es verpflichtend, einen I zu verwenden, und den betreffenden Kunden in der Zone A-D zu verrechnen lung betreffend Zuordnung, Erstellung und Anpassung von standardisierten Last 12.06, zuletzt geändert am 25.1.08). Er bezahlt die verbrauchte Arbeit und die ger g, deren Basis das arithmetische Mittel der im Abrechnungszeitraum (1 Jahr) m enen höchsten stündlichen Durchschnittsbelastung (12-Monats-Mittel) ist. Die ttelleistung ist die durch das Gesetz normierte Verrechnungseinheit, die allerding bei der Dimensionierung von Netzen keine Bedeutung hat, wo vielmehr die M entscheidend ist. Auch der im Gesetz angeführte Grundsatz der Kostenorientie der Tariffestlegung wird nur schlecht erreicht, da die monatliche Durchschnittsb diert, Kunden mit schlechter Auslastung (z. B. hohen Winterspitzen und geringe rauch) im Vergleich zu Kunden mit günstiger Fahrweise (d. h. geringen Leistung ontinuierliche Bandfahrweise) zu bevorzugen. Alle Kunden, die nicht in diese K n mit Leistungsmessung“ fallen, werden in der Zone 1-7 verrechnet (nicht-leist Kunden) und zahlen neben dem Arbeitsentgelt einen Pauschalbetrag, der neb estandteilen, wie der Verrechnung oder dem Störungsdienst in geringem Maße da es Leistungspreises der Zone A-F darstellt. Bei der Berechnung des Arbeitsprei ndsatz des Einkommenssteuertarifs angewendet Dies bedeutet, dass bei einem Ve pielsweise $17.000 \mathrm{kWh}$ die ersten $8.000 \mathrm{kWh}$ mit dem Tarif der Zone $1 \mathrm{zu}$ multip 
des Großversorgers ausschließlich in den Ebenen 2 und 3, im betrachteten Wiener Fall sind die ausgewählten Kundensegmente sogar nur in der Druckstufe $<6$ bar (Ebene 3).

Für die angeführte Kundenstruktur (siehe Abbildung 3) ergeben sich folgende Netzkosten im Wiener Netzgebiet: Das Gesamtentgelt beträgt rund 186 Mio. Euro, wobei der größte Anteil mit etwa 155 Mio. Euro dabei auf den Tarif für Arbeit entfällt (ein typisches Charakteristikum eines von Kleinkunden dominierten Netzgebiets).

\section{Energiebeschaffung}

Um Erdgaskunden das ganze Jahr hindurch zuverlässig versorgen zu können, müssen nicht nur Gaslieferverträge mit den großen Lieferanten (wie Gazprom oder Statoil) geschlossen werden, sondern darüber hinaus auch so genannte Speicher- oder Strukturierungsverträge mit Anbietern, die üblicherweise verbrauchsnah im Abnahmegebiet der Kunden angesiedelt sind. Dies ist deswegen bedeutsam, da das Gas von den Produktionsfeldern in einem mehr oder weniger konstanten Strom gefördert und transportiert wird, wohingegen der tatsächliche Verbrauch sehr stark temperaturabhängig ist und dementsprechend starken Schwankungen unterliegt. Ein üblicher bandförmiger Liefervertrag könnte mit den dort vereinbarten Bezugsflexibilitäten (zumeist nur wenige Prozent) niemals diese saisonalen Bedarfsänderungen abdecken. Der Lastgang des Versorgers über den Betrachtungszeitraum (in unserem Fall 8760 Stunden) wird durch die verbrauchte Energie („Arbeit“), die maximal benötigte Leistung sowie das erforderliche Speichervolumen beschrieben.

\section{Arbeit}

Die benötigte Energie, d. h. die von der Summe aller Einzelkunden im Jahr verbrauchte Gasmenge, muss in Form von Bezugsverträgen eingekauft werden; die benötigte Jahresenergiemenge wird im Falle Österreichs gewöhnlich zum überwiegenden Teil über Vollversorgungsverträge mit Vorlieferanten abgedeckt, die bei Kunden dieser Größe relativ flexible Bezugsmöglichkeiten bei schwankender Abgabe ermöglichen, d. h. großzügige Schwankungsbreiten bei den Jahresbezugsmengen bieten. Dies ist insbesondere bei großen Versorgern von entscheidender Bedeutung, da die jeweilige Abgabe in Abhängigkeit von den herrschenden Wetterbedingungen enorme Abweichungen von den Regel- bzw. Planwerten erreichen kann. Im besonders milden Jahr 2006/2007 kam es in Wien zu einem Einbruch der Abgabe um etwa 25 Prozent. Die Wahl der erforderlichen Bezugsmenge ist die grundlegende und wird in der Praxis oft in Anlehnung an den Bedarf eines temperaturbereinigten Regeljahres getroffen. Abbildung 3 entnehmen wir die Planabgabemenge unseres Versorgers von rund $13.100 \mathrm{GWh}$, die er beschaffungsseitig sichern muss, um seine Kunden versorgen zu können. Als Basis für die Verrechnung soll der durchschnittliche Importgaspreis (Statistik Austria 2009) für die letztverfügbaren zwölf Monate von April 2008 bis März 2009 herangezogen werden. Dies bedeutet bei einem Preis von circa 300 Euro/1000m³ bzw. 30 $\mathrm{Cent} / \mathrm{m}^{3}$ oder 2,7 Cent $/ \mathrm{kWh}$ und einem angenommenen Gewinnaufschlag des Vorlieferanten von 
0,1 Cent/kWh einen durchschnittlichen Jahresenergiepreis von 2,8 Cent/kWh. Daraus ergeben sich variable Energiebeschaffungskosten von rund 366,7 Mio. Euro.

\section{Speichervolumen}

Zudem ist die für die effektive Versorgung der Kunden erforderliche Strukturdienstleistung sicherzustellen: Da der Bezug des Erdgases großteils bandförmig erfolgt, der Verbrauch allerdings saisonal verteilt ist, muss Speichervolumen in geologischen Speicheranlagen angekauft werden, um Kunden bedarfsgerecht versorgen zu können. Nicht benötigte Bezugsmengen werden im Sommer eingespeist und in den starken Abnahmezeiten des Winters wieder entnommen. Das Speichervolumen erlaubt die Versorgung der in Wien besonders heizlastigen Kunden auch während der Hochlastzeiten bei gleichzeitig geringer Bandanlieferung. Die genaue Berechnung kann vertraglich auf unterschiedliche Weise erfolgen, hier soll das erforderliche Speichervolumen über die den Bandbezug übersteigende Menge errechnet werden. Diese Methode geht von der Überlegung aus, dass ein reiner Bandkunde mit einem Sommer-/ Winterverhältnis von 50:50 kein Speichervolumen benötigt, da sein gesamter Jahresverbrauch durch die eigentliche Gaslieferung abgedeckt werden kann. Sobald der Verbrauch im Winter höher wird, steigt der Volumenbedarf, um das Ungleichgewicht zwischen Sommer und Winter wieder ausgleichen zu können. Abbildung 3 zeigt den Volumenbedarf unterschiedlicher Kundensegmente. Im Bereich bis $8000 \mathrm{kWh}$ finden sich verhältnismäßig viele Kunden mit relativ ausgeglichenem Sommer-/Winterverhältnis, wie die in Wien besonders zahlreichen Kochgaskunden oder Warmwassernutzer. In den folgenden Stufen erkennt man das typische Heizverhalten mit deutlichem Überhang der Abnahmemenge im Winter und dementsprechend hohem benötigten Speichervolumen. Erst im (sehr kleinen) Bereich der Wiener Großkunden beginnt der Winteranteil wieder zu sinken, da Gas in diesem Segment verstärkt für die Produktion eingesetzt wird, die zumeist eine geringere Temperaturabhängigkeit aufweist. Die Berechnung des Entgelts für das Speichervolumen erfolgt in Anlehnung an jenes für die Speicherleistung und wird aus diesem Grund im nächsten Punkt „Speicherleistung“ behandelt.

\section{Speicherleistung}

Neben dem Ausgleich saisonaler Schwankungen müssen allerdings auch die stark schwankenden täglichen bzw. stündlichen Abgaben abgedeckt werden. Der Versorger muss insbesondere in der Lage sein, seine maximale stündliche Höchstlast (,stärkste Stunde“ - diese fällt üblicherweise in den Morgenstunden rund um 7-9 Uhr an den abgabestärksten Wintertagen an) abzudecken, was auf zwei Arten erfolgen kann: einerseits inkludiert der Einkauf der Energiemenge selbst eine zugehörige Grundleistung (Bandleistung - also die Leistung, die durch den Energiebezug selbst abgedeckt werden kann), die sich aus der Gesamtmenge/8760 Jahresstunden (inkl. etwaig vorhandener Flexibilitäten der Energiebezugsverträge) errechnet, darüber hinaus kann Speicherleistung in geologischen oder oberirdischen Anlagen zugekauft werden. Im Normalfall wird die Bandleistung alleine nicht ausreichen und der Zukauf von Speicherleistung nötig sein. Die Fest- 
legung dieser Gesamtleistung (Band- + Speicherleistung) ist von besonderer Bedeutung, da abhängig vom jeweiligen Vertragsmodell - bis zu 75 Prozent der gesamten Strukturkosten auf diesen Bereich entfallen.

In der Praxis sind verschiedene Methoden der Leistungsermittlung zu finden:

- Durchschnittsbildung: Mittels einfacher Methoden (wie etwa Durchschnittsbildung auf Basis der letzten verfügbaren Ist-Daten vergangener Jahre) erfolgt die Errechnung maximaler Stundenabgaben.

- Ableitung von technischen Norm-Auslegungsbedingungen bei der Berechnung der Heizlast von Gebäuden ausgehend von allgemein gültigen Richtlinien (ÖNORM H 7500 Verfahren zur Berechnung der Norm-Heizlast): Diese legen für die verschiedenen Bundesländer Norm-Auslegungstemperaturen fest (in Wien etwas weniger als $-13^{\circ} \mathrm{C}$ ). Auf Basis dieser Normtemperatur erfolgt die Festlegung der zu erwartenden maximalen Stundenabgabe.

- Regelabgabenschätzungen: Wiederum ausgehend von vorhandenen Ist-Daten erfolgt eine energiewirtschaftliche Bereinigung der Heizlast sowie die Berücksichtigung etwaiger Verluste an Konkurrenzenergien (im Raum Wien vor allem Fernwärme). Aus dieser Bewertung lassen sich Schlüsse auf die Maximalleistung ziehen.

- Risikoabschätzungen: Hier wird von bereits gegebenen, längerfristig stabilen, Nominierungsniveaus ausgegangen. In diesen Fällen werden die Auswirkungen von Leistungsniveauänderungen auf die Beschaffungskostensituation geschätzt, d. h. eine Verringerung der maximal eingekauften Spitzenleistung führt zu einem höheren Anfall zusätzlich benötigter variabler Energiemengen.

Oft kommt es zu Kombinationen dieser Vorgehensweisen, hier wird aus Gründen der Einfachheit die maximal nötige Speicherleistung aus der Betrachtung der vergangenen Jahre ermittelt und aus diesen vorhandenen Ist-Werten mittels einfacher Schätzung die bei $-12,4^{\circ}$ Celsius benötigte Leistung berechnet (Variante 1). Von dieser wird dann die Bandleistung subtrahiert, die sich aus der Gesamtbezugsmenge ableitet. Die Berechnung des für die Speicherleistung anzusetzenden Entgelts geht vom marktüblichen Preis für ein typisches Speicherprodukt aus. Als Richtwert soll hier der Tarif für Standarddienstleistungen, d. h. „,bundled services“, also Speicherleistung in Kombination mit Speichervolumen gewählt werden (OMV 2009). Im konkreten Beispiel wird als bestmöglicher Vergleichswert der fixe Tarif (10-Jahres-Kontrakt) gewählt, der an fixe Zeiträume für die Einpressung (üblicherweise Sommer) bzw. Entnahme (Winter) gebunden ist. Als angenommener Jahresleistungspreis ergibt sich somit ungefähr $128 \mathrm{EURO} / \mathrm{m}^{3} / \mathrm{h}$ auf der Basis des Jahres 2009. Die genaue Gewichtung der Speicherleistungs- und Speichervolumenanteile des Gesamtentgelts ist nicht eindeutig ermittelbar; ausgehend von den praktischen Erfahrungen aus der Zeit vor der Liberalisierung des Gasmarkts wird der Leistungsanteil vereinfachend mit 75 Prozent vom Gesamtentgelt angesetzt, das bedeutet $96 \mathrm{EUR} / \mathrm{m}^{3} / \mathrm{h}$ - umgerechnet $8,6 \mathrm{EUR} / \mathrm{kWh} /$ h. Bei der schon zuvor erwähnten Speicherleistung von 3.300 MW errechnen sich Energieleistungskosten von ungefähr 28,6 Mio. Euro.

Bei der Berechnung des Volumenentgelts wird der grundsätzlichen Vorgehensweise gefolgt, die soeben bei der Berechnung des Leistungsentgelts angewendet wurde - es werden also 25 Prozent des OMV-Tarifs für Standarddienstleistungen als Volumenpreis angesetzt, das bedeutet $32 \mathrm{EUR} /$ 
$\mathrm{m}^{3} / \mathrm{h}$ - oder umgerechnet 2,9 EUR/kWh/h. ${ }^{2}$ Die Kosten für das Volumen werden also über die Leistung angenähert, was in der Praxis auch sinnvoll ist, da nur ein verfügbares Volumen in der Speicheranlage auch die nachfolgende Ausspeicherung ermöglicht. Der Wert des gesamten Einlagerungsvolumens für die Wiener Kunden von etwa 3.400 GWh wird über die benötigte Speicherleistung von 3.300 MW hergeleitet, das sind somit 9,5 Mio. Euro.

\section{Energiebezugsnebenkosten}

Neben den soeben beschriebenen Hauptelementen bei der Beschaffung von Erdgas müssen auch noch Kosten abgedeckt werden, die sich mit der Etablierung des Bilanzgruppensystems im österreichischen Markt ergeben haben. Alle Kostenansätze sind in den Marktregeln für den Erdgasmarkt zu finden (E-Control 2009):

- Bilanzgruppenmitgliedschaft: Im Marktsystem muss jeder Zählpunkt Mitglied einer Bilanzgruppe sein, um zu gewährleisten, dass auch alle eingespeisten und entnommenen Mengen systemtechnisch bilanziert werden können. Während Kraftwerke oder andere Großkunden üblicherweise ihre Mengenprognosen eigenständig erstellen und somit unmittelbare Mitglieder einer Bilanzgruppe sein können, wird die große Zahl der Kleinkunden von Versorgern zusammengefasst und gegenüber der Bilanzgruppe ,vertreten“ (mittelbare Bilanzgruppenmitgliedschaft). Die Abrechnung erfolgt über die verbrauchte Arbeit, das Entgelt beträgt: 2,12 ct/ MWh bzw. 280.000 Euro.

- Fahrplanerstellung (Mengennominierung) und die damit zusammenhängende Abdeckung des Ausgleichsenergierisikos: Im Bilanzgruppensystem erfolgt die kurzfristige Mengennominierung auf Basis von Fahrplänen, die am Vortag (day-ahead) und nach Bedarf mit zwei Stunden Vorlaufzeit am eigentlichen Verbrauchstag (intra-day) gesendet werden müssen. Abweichungen der angemeldeten Fahrplanmengen zu den tatsächlichen Ist-Mengen der einzelnen Stunden werden mit Ausgleichsenergie glattgestellt und die angefallenen Ausgleichsenergiekosten den Verursachern verrechnet (im Fall von Überlieferungen gutgeschrieben). Öffentlich verfügbare Marktpreise sind auf Grund der geringen Anbieterzahl derzeit noch nicht vorhanden. Ausgehend von praktischen Erfahrungen wird ein verbrauchsabhängiges Entgelt von 12,5 ct/MWh angenommen, was 1.650.000 Euro entspricht.

- Clearinggebühren: Für die gesamte im Jahr verbrauchte Energiemenge des Versorgers sind als Entgelt für die Ausgleichsenergieermittlung und -verwaltung an die Verrechnungsstelle (Bilanzgruppenkoordinator) Clearinggebühren zu entrichten. Der aktuell gültige Entgeltsatz beträgt 3,99 ct/MWh oder rund 525.000 Euro.

- Messentgelt für die Einspeisung von Erdgas aus dem Import, aus Produktion sowie aus den Speicheranlagen: Neben den direkt dem Endverbraucher verrechneten Messkosten, fallen auch bei der Messung eingespeister Mengen in die Regelzone Kosten an, die von den Bilanzgruppenverantwortlichen nach verbrauchter Menge an die Versorger weitergegeben werden. Das Messentgelt beträgt: 2,88 ct/MWh oder ca. 380.000 Euro.

\footnotetext{
$21 \mathrm{~m}^{3}$ entspricht hier $11,14 \mathrm{kWh}$.
} 
- Sicherheitsleistungen: Um als Teilnehmer im Erdgasmarkt tatsächlich zugelassen zu werden, sind die Bilanzgruppenverantwortlichen zur Stellung von Sicherheiten verpflichtet, die zur Deckung etwaiger finanzieller Folgen aus Zahlungsverzug dienen. Die Höhe der Sicherheiten richtet sich neben der Bonitätsbewertung vor allem nach dem Monatsumsatz, der ja den maximal möglichen finanziellen Ausfall hauptsächlich beeinflusst. Auf die genaue Herleitung soll in diesem Rahmen verzichtet werden; bei einem Versorger dieser Größe kann von einem Jahresbeitrag von etwa 25.000 Euro ausgegangen werden.

\section{Sonstige Kosteneinflussfaktoren}

\section{Messkosten}

Für die Beistellung der zur Gasmengenmessung erforderlichen Messeinrichtungen durch den Verteilernetzbetreiber hat der Kunde ein Entgelt zu entrichten. Dieses umfasst neben der eigentlichen Zurverfügungstellung des Zählers auch den Betrieb, die Eichung und die Zählerdatenauslesung, somit auch die Verwaltung und Verrechnung aller zählerrelevanten Daten und Auswertungen. Wie Abbildung 5 zeigt, ist auf Grund des klein strukturierten Wiener Erdgaskundenmarktes eine massive Häufung von kleinen Gaszählern der Größen G4 und G6 zu finden. Die leicht unterschiedliche Anzahl der eingesetzten Gaszähler und der Kunden im Versorgungsbereich erklärt sich dadurch, dass insbesondere Großkunden auch mehrere Zähler im Einsatz haben können. Zu den Messkosten gehören im weiteren Sinne auch die Kosten für die Ablesung, die allerdings getrennt auszuweisen sind. Für den jährlich abzulesenden Kleinkunden betragen diese vier Euro je Jahr, während bei den monatlich verrechneten, größeren Kunden acht Euro pro Monat anfallen (Abbildung 5). 


\section{Überblick über die Gesamtkostensituation}

or errechneten Teilkostenkomponenten (inklusive des Gewinnzuschlages des $\mathrm{H}$ ers) sollen überblicksmäßig nochmals dargestellt und zusammengefasst werden Vie bereits oben angeführt, wurden die Positionen Marge (10 Prozent auf die Sum den Kosten), sowie Marketing, Personal etc. (Fixbetrag 20 Mio. Euro) auf Gru a Bedeutung in dieser vorwiegend energiewirtschaftlichen Betrachtung nur mit ngen berücksichtigt (Abbildungen 6 und 7).

\section{amtkostenübersicht Marge)}

\begin{tabular}{lr} 
& \\
rtkosten & 186,0 \\
\hline sten & 17,4 \\
& 203,4 \\
\hline & \\
\hline ezug & 366,7 \\
\hline rvolumen & 9,5 \\
rleistung & 28,6 \\
stem & 2,8 \\
\hline ersonal, ...) & 20,0 \\
\hline & 42,8 \\
& 470,4 \\
\hline
\end{tabular}

\section{\& Abgaben}

hsabgabe

bgabe

43,0

77,8

teuer

158,9

279,8

953,5
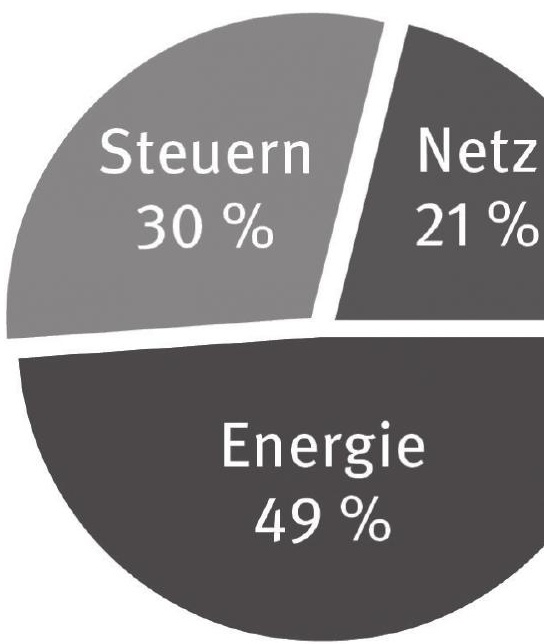

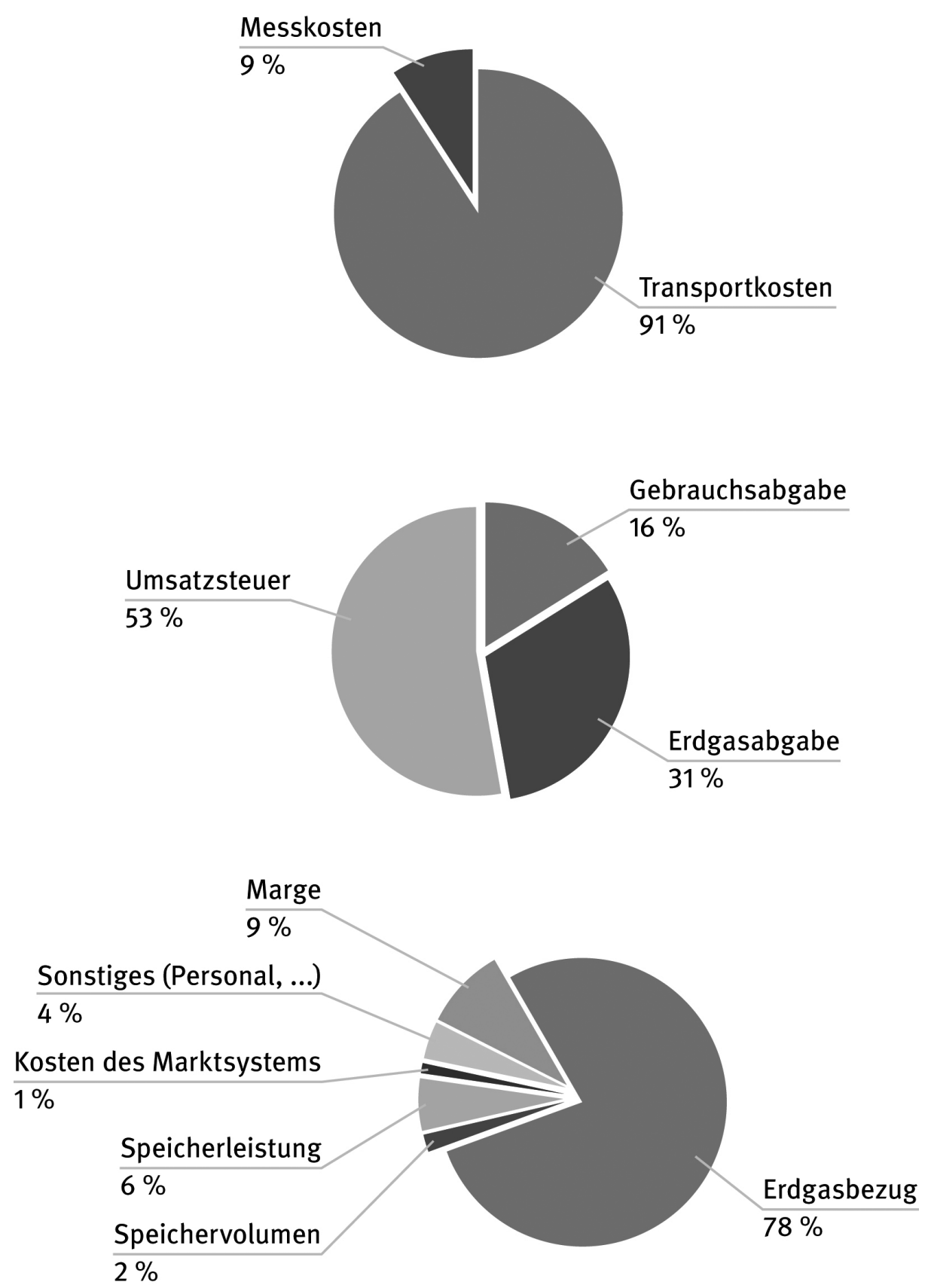

Abb. 7: Aufteilung der Netz-, Energie- und Steueranteile

Quelle: Eigene Darstellung 


\section{Schlussfolgerung}

Die Liberalisierung des Erdgasmarktes in Österreich hat zu einer weitreichenden Auftrennung der Vertriebs- und Netzaufgaben geführt. Trotz dieser Teilung der früher in einer Hand gelegenen Aufgaben kann sich der Vertrieb dem Kunden gegenüber nicht auf die Rolle des reinen Energielieferanten zurückziehen. Zwar sind neben den Steuern und Abgaben auch die Kostenkomponenten für die Netznutzung und die Messdienstleistung aus seiner Sicht unbeeinflussbar, dennoch steht er aus Sicht des Kunden - anders als der monopolistische Netzbetreiber - oftmals stellvertretend für die gesamte Erdgaslieferung. Aus diesem Grund muss der Vertrieb als Energiedienstleister stets den Blick auf die, für die Kunden relevanten, Gesamtkosten wahren, um gegenüber anderen Erdgasanbietern, aber auch den klassischen Konkurrenzenergien, erfolgreich zu bleiben. Dieser Umstand ist jedenfalls aus kommunikationspolitischer Hinsicht entsprechend zu berücksichtigen, da die meisten Kunden nach wie vor die Auswirkungen der Liberalisierung und der damit einhergehenden Funktionsteilung kaum zur Kenntnis genommen haben. Zu diesem grundlegenden Verständniswechsel gehört auch ein verstärktes Engagement im Bereich innovativer Neuentwicklungen im Gasbereich (beispielsweise Smart Metering). Diese werden üblicherweise vordringlich vom Netzbetreiber betreut, was zwar aus dem Blickwinkel technologischer Umsetzbarkeit sinnvoll ist, die Formulierung kundenorientierter Produktideen sollte allerdings in der Verantwortlichkeit des Energievertriebs liegen.

Abstract

Peter Deschkan; Consideration of the relevant cost components in the liberalized Austrian natural gas markets from the perspective of a Viennese energy service provider

Balancing group system; Cost analysis; Natural gas market; Natural gas supplier; Liberalized market; Unbundling

Following the complete opening of the Austrian natural gas markets in October 2002 the formerly dominating local incumbents had to split up to meet the stringent unbundling requirements. During the last few years this process has effectively led to separated gas grid operating and energy supply companies. This newly won autonomy requires a changed view on the relevant cost components and the market position as compared with competitors in the natural gas market and substitute energies, like oil, district heating or alternative energy sources. To remain successful in the liberalized market the focus of the supplier still has to lie on the total price for the final 
customer, although he is no longer capable to influence all of its components. In the following article all cost components will be shown for the specific case of the Viennese natural gas supply.

\section{Literaturverzeichnis}

AGGM (2009), Statistik Netzzugang, http://www.aggm.at/jart/prj3/aggm/main.jart?rel=de\&contentid=1170174614305 (Zugriff: 1.1.2010).

Crastan, Valentin (2009), Elektrische Energieversorgung 2, 2.Auflage, Berlin-Heidelberg.

E-Control (2009), Marktregeln für den österreichischen Erdgasmarkt, http://www.e-control.at/de/marktteilnehmer/ gas/marktregeln (Zugriff 1.1.2010).

OMV (2009), Tarif für Speicherdienstleistung, http://www.omv.com (Zugriff 1.1.2010).

Wiedmann, Klaus-Peter, Jörg Hennings und Thomas Kilian (2005), Wechselbereitschaft privater Endkunden als zentrale Herausforderung im Erdgasmarketing, in: Der Markt, Vol. 44, Nr. 1, März 2005, S. 44-54. 